\title{
Emphatically Stressed Demonstratives
}

\author{
Charley Beller
}

Johns Hopkins University

\section{Introduction}

This paper concerns the linguistic packaging of emotive content. The particular phenomenon of interest appears to be an interaction between this linguistic packaging of emotive content and the standard workings of anaphoricity. I provide an explanation for why stressed demonstrative nominals (SDNs) can corefer but not covary.

Stress and coreference: The effects of stress on the resolution of pronouns have been familiar since at least Akmajian and Jackendoff (1970). The example below shows the symmetry of these stress effects for pronouns, definite descriptions, and demonstrative determiners. When they are not stressed they can be easily coreferential with a discourse antecedent, while the stressed variants are not as easily coreferential in the same context.
Jack $_{i}$ kissed Mary.
a. I hate $\operatorname{him}_{i}$
b. I hate $\mathrm{HIM}_{\# i / j}$
c. I hate the creep $_{i}$
d. I hate the CREEP $\# i / j$
e. I hate that $\mathrm{creep}_{i}$
f. I hate THAT creep $_{\# i / j}$

What has been largely unremarked upon (though see Beller to appear) is that the symmetry breaks when the nominal complement of a demonstrative determiner bears stress. In (2) we see that even when the nominal in a demonstrative DP bears stress (i.e. even when it is a stressed demonstrative nominal), the DP can still easily corefer with a discourse antecedent.

$$
\mathrm{Jack}_{i} \text { kissed Mary. I hate that } \mathrm{JERK}_{i} \text {. }
$$

Stress and binding: A similar stress effect shows up when an anaphoric element is in the scope of a quantifier. When pronouns, definite descriptions, and demonstrative determiners are unstressed the reference of the DPs can covary with the quantifier.

(3) Every senator i $_{i}$ was so dishonest that ...
a. he $e_{i}$ accepted a bribe.
b. the creep $i$ accepted a bribe.
c. that senator ${ }_{i}$ accepted a bribe.

When these elements are stressed a covarying reading is much more difficult to obtain. Thus the DPs in (4) are all interpreted as being disjoint from the quantified subject.

(4) Every senator ${ }_{i}$ was so dishonest that ...
a. $\mathrm{HE}_{* i / j}$ accepted a bribe.
b. the $\mathrm{CREEP}_{* i / j}$ accepted a bribe.
c. THAT senator ${ }_{* i / j}$ accepted a bribe. 
Unlike in the coreferential case stressed demonstrative nominals pattern like the other stressbearing DPs receiving a disjoint interpretation, (5).

(5) Every senator ${ }_{i}$ was so dishonest that that CREEP $_{* i / j}$ accepted a bribe.

Stress and donkey-anaphora: Donkey sentences like those in (6) have a chain of anaphoric dependencies. The donkey anaphor itself (e.g. him in the a. continuation) has a coreferencelike dependency on the indefinite a senator in the relative clause. The reference of that indefinite covaries with the quantified subject. As before these dependencies are fine when the anaphoric element does not bear stress, (6).

(6) Every intern who works for a senator ${ }_{i} \ldots$
a. ends up disliking $\operatorname{him}_{i}$.
b. ends up disliking the $\operatorname{creep}_{i}$.
c. ends up disliking that $\mathrm{creep}_{i}$.

And if a pronoun, definite description, or demonstrative determiner bears stress a disjoint reading is heavily favored. This is understandable since the lower, coreference-like dependency, should be susceptible to the same disruption as in the coreferential cases above.

(7) Every intern who works for a senator ${ }_{i} \ldots$
a. ends up disliking $\mathrm{HIM}_{* i / j}$.
b. ends up disliking the $\mathrm{CREEP}_{* i / j}$.
c. ends up disliking THAT creep $_{* i / j}$.

With an SDN the higher dependency is disrupted, disallowing a covarying reading, (8). But the lower dependency can be maintained so long as the indefinite antecedent a senator takes wide scope over the universal quantifier, as in (9). In this reading all the interns in question work for the same senator.

(8) Every intern who works for a senator $i$ ends up disliking that $\mathrm{CREEP}_{i}$.

(9) $\quad \exists x . x$ a senator. $\forall y$. $y$ an intern \& $y$ works-for $x \rightarrow y$ ends-up-disliking $x$.]

Interestingly the same set of readings is available when the nominal is modified by an expressive adjective, as in (10).

(10) Every intern who works for a senator ends up disliking that $\mathrm{f}$ !\#@ing creep.

The analysis I pursue takes seriously the similarity in interpretation between (8) and (10). The core of the proposal is that the emphatic stress on SDNs is represented as an expressive adjective. This allows SDNs to be subsumed under the theory of expressive meaning presented in Potts (2007). On that theory emotional attitudes are represented by a set of expressive indices which are manipulated in a specific way by expressive adjectives. After 
introducing Potts's machinery the remainder of the analysis involves demonstrating that the mechanism behind manipulating expressive indices do not admit covarying interpretations.

\section{Emphatic stress as an expressive element}

Here I present a somewhat simplified version of Potts's framework. A discourse context is a tuple $c$ containing specifications of the speaker, the time, the world, and a set $E$ of expressive indices. These indices are triples of the form $\langle a \mathcal{I} b\rangle$, where $a$ and $b$ are individuals and $\mathcal{I}$ is an interval between -1 and +1 . For any two individuals in $D_{e}$ there is at most a single expressive index, representing the attitude that the first individual holds toward the second. The interval represents the content of that attitude. The polarity on the interval reflecting the polarity of the emotional attitude and the width of the interval indicates the strength of the attitude.

Expressive elements like the adjective damn are context shifting operators that change a context by update an expressive index by narrowing its interval. The ontology is expanded to include a basic type for expressives, $\epsilon$, and a corresponding domain, $D_{\epsilon}$. Complex expressive types all have the form $\langle\sigma, \epsilon\rangle$, where $\sigma$ is a descriptive type. Expressive elements use their descriptive arguments to identify which indices to overwrite and then return those arguments under the appropriately shifted context.

As foreshadowed above the denotation I treat the emphatic stress on SDNs as the surface realization of an abstract morpheme $E M P H$, which has the denotation shown in (11).

$$
\llbracket \mathbf{E M P H} \rrbracket^{c}=\lambda x_{e} . \mathrm{c}^{\prime} \text { such that }
$$
i. $\left\langle\mathrm{S} \mathcal{I} \llbracket \mathbf{x} \rrbracket^{c}\right\rangle \in \mathrm{c}$
ii. $\left\langle\mathrm{S} \mathcal{I}^{\prime} \llbracket \mathbf{x} \rrbracket^{c}\right\rangle \in \mathrm{c}^{\prime}$
iii. $\mathcal{I}^{\prime} \sqsubseteq \mathcal{I}$
iv. $\mathcal{I}^{\prime} \sqsubseteq[-1,0]$

EMPH takes an individual $x$ and shifts the context from that provided by the $c$ parameter to output context $c^{\prime}$ such that the expressive index representing the emotional attitude of the speaker toward the individual $x$ is narrower than in the input context, and is negative. Expressive adjectives like damn have a very similar denotation, though possibly a different specification for the superinterval in line (iv).

To illustrate the effect of $E M P H$, consider a discourse in which the unstressed demonstrative DP that jerk has the evaluation in (12). In such a discourse the use of the SDN that JERK would instead have the structure and evaluation shown in (13).

$$
\begin{aligned}
& \llbracket \text { that }_{\text {jerk }} \rrbracket^{c}=\text { Jack } \\
& \text { where } E \in c=\{\ldots\langle\mathrm{S},[-1,1], \text { Jack }\rangle \ldots\} \\
& \llbracket \mathbf{E M P H} \rrbracket^{c}\left(\llbracket \text { that jerk } \rrbracket^{c}\right)=\llbracket \text { that jerk }_{i} \rrbracket^{c^{\prime}} \\
& \text { where } E^{\prime} \in c^{\prime}=\{\ldots\langle\mathrm{S},[-1,0], \text { Jack }\rangle \ldots\}
\end{aligned}
$$

\section{Context shifting with a covarying argument}

The illustration in (13) showed the effect of EMPH when the demonstrative DP is coreferential. What happens when the DP covaries? There are two strategies that may be employed to compute the output context: (i) a serial context-shifting strategy and (ii) a parallel context-shifting strategy. In the following I argue that the serial strategy results 
in an output context indistinguishable from that generated by a coreferential DP. The parallel strategy produces an output context with the intuitively appropriate set of expressive indices, but this strategy is unavailable to singular indefinites due to a feature mismatch.

Serial context-shifting: The serial strategy involves iterating through potential values of the covarying argument. For each value of the argument EMPH returns an output context. This context shifting mechanism has no memory for intermediate states and so the input to $E M P H$ is the initial context $c$ in each instance of its application.

Thus there is no chain of transitions $\mathrm{c} \rightarrow \mathrm{c}^{\prime} \rightarrow \mathrm{c}^{\prime \prime} \rightarrow \mathrm{c}^{\prime \prime \prime}$. Instead each successive change overwrites the last. $\mathrm{c} \rightarrow \mathrm{c}^{\prime}$ is overwritten by $\mathrm{c} \rightarrow \mathrm{c}^{\prime \prime}$ which is overwritten in turn by $\mathrm{c} \rightarrow \mathrm{c}^{\prime \prime \prime}$. The final output is a context that differs from $c$ by at most a single expressive index, just as in the coreferential case.

Parallel context-shifting: The parallel strategy involves aggregating the possible values of the covarying argument and feeding the set of values to the context-shifting operation. This will yield a set of output contexts, $C^{\text {out }}$. Each output context $c^{\prime} \in C^{\text {out }}$ will differ from $c$ by a single index, so the set can be reduced to a single output context by an integration procedure like that in (14).

Context integration: For all $c^{\prime}$ and $c^{\prime \prime} \in C^{\text {out }}, \sqcup_{C}\left(c^{\prime}, c^{\prime \prime}\right)=c^{\prime \prime \prime}$ such that:

For any corresponding expressive indices $e_{a b}^{\prime} \in c^{\prime}$, and $e_{a b}^{\prime \prime} \in c^{\prime \prime}$

If interval $I_{a b}^{\prime} \sqsubseteq I_{a b}^{\prime \prime}$ :

Then $e_{a b}^{\prime}$ is in $E^{\prime \prime \prime} \in c^{\prime \prime \prime}$.

Else: $e_{a b}^{\prime \prime}$ is in $E^{\prime \prime \prime} \in c^{\prime \prime \prime}$.

Parallel context shifting and plurality: Parallel context shifting is necessary for plural SDNs as in (15). These plural SDNs convey only a distributive negativity in which the speaker disapproves of each of the atomic individuals.

Dave and John said they were sick but Margot saw those BASTARDS at the theater.

I propose that this relationship goes both ways: Plural expressives require the parallel strategy and the parallel strategy requires a plurality feature. This feature may be licensed by the aggregation inherent in universal quantifiers. When the anaphoric element is a singular demonstrative a feature mismatch will crash the derivation. As such SDNs in singular demonstrative DPs can implement only the serial context-shifting strategy.

Alternatively singular SDNs can have a referential antecedent. In donkey-sentences this antecedent can be extra-sentential, or the intra-sentential indefinite antecedent can scope high. In the scope of a quantifier there is no intra-sentential antecedent so the SDN must have a disjoint interpretation.

Akmajian, A., and R. Jackendoff. 1970. Coreferentiality and stress. Linguistic Inquiry 1:124-126.

Beller, C. to appear. Emotive content, scope, and donkey demonstratives. In Proceedings of the Western Conference on Linguistics (WECOL) 2011.

Potts, C. 2007. The expressive dimension. Theoretical Linguistics 33:165-198. 